\title{
TOP-DESIGNS IN THE CATEGORY OF FORT SPACES
}

\author{
MEHRNAZ POURATTAR, FATEMAH AYATOLLAH ZADEH SHIRAZI
}

Abstract. In infinite topological Fort space $X$, for nonempty subsets $C, D$ of $X$ in the following text we answer to this question "Is there any $\lambda$ and Top-design $C-(X, D, \lambda)$ of type $i$ ?" for $i=1,2,3,4$. We prove there exist $\lambda$ and $C-(X, D, \lambda)$, Top-design of type 2 (resp. type 4 ) if and only if $C$ can be embedded into $D$.

2010 Mathematics Subject Classification: 54, 05B05

Keywords: Fort space, Generalized-design, Top-design.

\section{INTRODUCTION}

Suppose $S$ is a finite set with $n \geq 2$ elements (so $S$ is an $n$-set) and $\mathbb{A}$ is a collection of $k$-subsets of $S$ such that each $t$-subset of $S$ occurs exactly in $\lambda$ elements of $\mathbb{A}$, then $\mathbb{A}$ is favorit and well studied traditional $t-(n, k, \lambda)$ combinatorial design $(t<n$ and $\lambda \geq 1$ ) (see [1,3]). However these finite traditional designs has been generalized in "infinite designs" in [3], also generalized designs have been introduced for the first time in [2] as a generalization of combinatorial designs in different mathematical categories like category of well-ordered sets, topological spaces, etc.. We use term Top-design when our reference category is the category of topological spaces.

Using the same notations as in [2], in topological space $X$ for nonempty subsets $C, D$ of $X$, nonzero cardinal number $\lambda$ and collection $\mathbb{A}$ of subsets of $X$ using statements (where by $S \approx T$ we mean $S$ and $T$ are homeomorphic spaces):

I. $\forall B \in \mathbb{A}(B \approx D)$

II. $\forall B \in \mathbb{A} \quad(B \approx D \wedge X \backslash B \approx X \backslash D)$

III. $\forall E \subseteq X \quad(E \approx C \Rightarrow \operatorname{card}(\{B \in \mathbb{A}: E \subseteq B\})=\lambda)$

IV. $\forall E \subseteq X \quad((E \approx C \wedge X \backslash E \approx X \backslash C) \Rightarrow \operatorname{card}(\{B \in \mathbb{A}: E \subseteq B\})=\lambda)$,

we say $\mathbb{A}$ is a :

- $C-(X, D, \lambda)$ Top-design of type 1 , if (II) and (III)

- $C-(X, D, \lambda)$ Top-design of type 2 , if (I) and (III)

- $C-(X, D, \lambda)$ Top-design of type 3 , if (II) and (IV)

- $C-(X, D, \lambda)$ Top-design of type 4 , if (I) and (IV)

Let's mention that if $b \in X$, equip $X$ with topology $\{U \subseteq X: b \notin U \vee(X \backslash U$ is finite) $\}$, then we say $X$ is a Fort space with particular point $b$ [4, Counterexample 24]. One may find counterexamples regarding $C-\left(\left\{\frac{1}{n}: n \geq 1\right\} \cup\{0\}, D, \lambda\right)$ Topdesigns in [2], note to the fact that $\left\{\frac{1}{n}: n \geq 1\right\} \cup\{0\}$ (with induced topology of $\mathbb{R})$ is an infinite countable Fort space, leads us to study other types of infinite Fort spaces in the approach of Top-designs.

Note 1.1. Two Fort spaces are homeomorph if and only if they are in one-to-one correspondence. Moreover in Fort space $X$ with particular point $b$ infinite subset $Y$ 
of $X$ as subspace topology has Fort topology if and only if $b \in Y$ (all finite subsets of $X$ are finite discrete spaces and carry Fort topology structure).

Convention 1.2. In the following text suppose $X$ is an infinite Fort space with the particular point $b$.

\section{Results in Top-Designs on $X$}

In this section we study the existence of $C-(X, D, \lambda)$ for different $C \mathrm{~s}$ and $D \mathrm{~s}$.

Lemma 2.1. For $U, V \subseteq X$ with $U \approx V$ and $X \backslash U \approx X \backslash V$ we have:

1. $b \in U$ if and only if $b \in V$ (i.e., $U \cap\{b\}=V \cap\{b\}$ ),

2. for infinite $U$ with $\operatorname{card}(U)<\operatorname{card}(X)$ and $H \subseteq X$ we have $U \approx H$ and $X \backslash U \approx X \backslash H$ if and only if $\operatorname{card}(U)=\operatorname{card}(H)$ and $U \cap\{b\}=H \cap\{b\}$.

Proof. 1) First suppose $U$ is infinite, so $V$ is infinite too. Since $b$ is the unique limit point of any infinite subset of $X, U$ contains a limit point if and only if $b \in U$ on the other hand $U$ contains a limit point if and only if $V$ contains a limit point which means $b \in V$ in its turn.

Now suppose $U$ is finite, thus $X \backslash U$ is infinite and using a similar method described above, we have $b \in X \backslash U$ if and only if $b \in X \backslash V$ which completes the proof.

2) Suppose $\operatorname{card}(U)=\operatorname{card}(H)$ and $U \cap\{b\}=H \cap\{b\}$, then $\operatorname{card}(U \backslash\{b\})=$ $\operatorname{card}(H \backslash\{b\})$, thus there exists bijection $f: U \backslash\{b\} \rightarrow H \backslash\{b\}$. If $b \notin U$, then $b \notin H$ and $f: U \backslash\{b\}=U \rightarrow H \backslash\{b\}=H$ is a homeomorphism (of discrete spaces) too. If $b \in U$, then $b \in H$ too and $\tilde{f}: U \rightarrow H$ with $\tilde{f} \uparrow_{U \backslash\{b\}}=f$ and $\tilde{f}(b)=b$ is a homeomorphism of infinite Fort spaces ( $U$ and $H$ with particular point $b$ ). So $U \approx H$.

On the other hand if $\operatorname{card}(U)=\operatorname{card}(H)<\operatorname{card}(X)$, then $\operatorname{card}(X \backslash U)=\operatorname{card}(X \backslash$ $H)=\operatorname{card}(X)$. Also if $U \cap\{b\}=H \cap\{b\}$, then $(X \backslash U) \cap\{b\}=(X \backslash H) \cap\{b\}$. So if $\operatorname{card}(U)=\operatorname{card}(H)<\operatorname{card}(X)$ and $U \cap\{b\}=H \cap\{b\}$, then $\operatorname{card}(X \backslash U)=$ $\operatorname{card}(X \backslash H)$ and $(X \backslash U) \cap\{b\}=(X \backslash H) \cap\{b\}$ which shows $X \backslash U \approx X \backslash H$ by the above argument.

Use item (1) to complete the proof of (2).

Note that if there exists a $C-(X, D, \lambda)$, Top-design of type $i$, then there exists $U \approx D$ with $C \subseteq U$, so $\operatorname{card}(C) \leq \operatorname{card}(U)=\operatorname{card}(D)$. Therefore $\operatorname{card}(C)=$ $\min (\operatorname{card}(D), \operatorname{card}(C)) \leq \operatorname{card}(X)$.

Theorem 2.2. Regarding 1st type of Top-designs for nonempty subsets $C, D$ of $X$ we have:

a. suppose $b \notin C \cup D$ :

a. if $C$ is finite, then there is not any $C-(X, D, \lambda)$, Top-design of type 1 ,

$\mathrm{a}_{2}$. if $C$ is infinite and $\operatorname{card}(C)<\operatorname{card}(X)$, then there exist $\lambda$ and a $C-(X, D, \lambda)$

Top-design of type 1 ,

a3. if $\operatorname{card}(C)=\operatorname{card}(D)=\operatorname{card}(X)$, then there exist $\lambda$ and a $C-(X, D, \lambda)$

Top-design of type 1 if and only if $D=X \backslash\{b\}$,

b. if $b \in C \backslash D$, then there is not any $C-(X, D, \lambda)$, Top-design of type 1 ,

c. suppose $b \in D$ :

$\mathrm{c}_{1}$. for finite $C$ there exist $\lambda$ and a $C-(X, D, \lambda)$ Top-design of type 1 if and only if $\operatorname{card}(C)+2 \leq \operatorname{card}(D)$, 
$\mathrm{c}_{2}$. if $C$ is infinite and $\operatorname{card}(C)=\min (\operatorname{card}(D), \operatorname{card}(C))<\operatorname{card}(X)$, then there exist $\lambda$ and a $C-(X, D, \lambda)$ Top-design of type 1 ,

$\mathrm{c}_{3}$. if $\operatorname{card}(C)=\operatorname{card}(D)=\operatorname{card}(X)$, then there exist $\lambda$ and a $C-(X, D, \lambda)$

Top-design of type 1 if and only if $D=X$.

Proof. Let $\mathbb{W}=\{E \subseteq X: E \approx D \wedge X \backslash E \approx X \backslash D\}$. By item (1) in Lemma 2.1, it's evident that $b \in D$ if and only if $b \in \bigcup \mathbb{W}$ (resp. $b \in \bigcap \mathbb{W}$ ).

$\underline{a_{1}}$ ) Choose $k \in C$, if $\mathbb{A}$ is a $C-(X, D, \lambda)$, Top-design of type 1 , then $\mathbb{A} \subseteq \mathbb{W}$ and $\mathbb{A}$ is a $(C \backslash\{k\}) \cup\{b\}-(X, D, \lambda)$, Top-design of type 1 too, which is a contradiction since $b \notin \bigcup \mathbb{W}$.

$\left.a_{2}\right)$ We have the following sub-cases:

- $\operatorname{card}(C) \leq \operatorname{card}(D)<\operatorname{card}(X)$. In this case by item (2) in Lemma 2.1 we have $\mathbb{W}=\{E \subseteq X \backslash\{b\}: \operatorname{card}(E)=\operatorname{card}(D)\}$. Using $\operatorname{card}(X \backslash\{b\})=\operatorname{card}(X \backslash(C \cup\{b\}))$

for $\mathbb{W}^{\prime}=\{E \subseteq X \backslash(C \cup\{b\}): \operatorname{card}(E)=\operatorname{card}(D)\}$ we have $\operatorname{card}(\mathbb{W})=\operatorname{card}\left(\mathbb{W}^{\prime}\right)$. It's evident that $\underset{E \mapsto E \cup C}{\mathbb{W}} \rightarrow \mathbb{W}$ is one-to-one, so card $\left(\mathbb{W}^{\prime}\right) \leq \operatorname{card}(\{F \in \mathbb{W}: C \subseteq F\}) \leq$ $\operatorname{card}(\mathbb{W})$. Thus $\operatorname{card}(\{F \in \mathbb{W}: C \subseteq F\})=\operatorname{card}(\mathbb{W})$. Since $C$ is infinite and $b \notin C$, for all subset $E$ of $X$ with $C \approx E$ we have $\operatorname{card}(C)=\operatorname{card}(E)$ and $b \notin E$, so by a similar method described for $C$ we have $\operatorname{card}(\{F \in \mathbb{W}: E \subseteq F\})=\operatorname{card}(\mathbb{W})$. Hence $\mathbb{W}$ is a $C-(X, D, \operatorname{card}(\mathbb{W}))$ Top-design of type 1 .

- $\operatorname{card}(C)<\operatorname{card}(D)=\operatorname{card}(X)$. In this case by Lemma 2.1, W $=\{E \subseteq X \backslash\{b\}$ : $\operatorname{card}(E)=\operatorname{card}(D) \wedge \operatorname{card}(X \backslash E)=\operatorname{card}(X \backslash D)\}$. Since $\operatorname{card}(C)<\operatorname{card}(D)$ and $C, D$ carry discrete topologies thus $C$ can be embedded in $D$ and without any loss of generality we may suppose $C \subseteq D$. By infiniteness of $D$, at least one of the sets $D \backslash C$ or $C$ is infinite and

$$
\begin{aligned}
\operatorname{card}(C) & <\operatorname{card}(X)=\operatorname{card}(D) \\
& =\operatorname{card}(C)+\operatorname{card}(D \backslash C) \\
& =\max (\operatorname{card}(C), \operatorname{card}(D \backslash C))
\end{aligned}
$$

so we have $\max (\operatorname{card}(C), \operatorname{card}(D \backslash C))=\operatorname{card}(D \backslash C)=\operatorname{card}(X)$. Since $2 \operatorname{card}(D \backslash$ $C)=\operatorname{card}(D \backslash C)$, we may choose $H \subseteq D \backslash C$ with

$$
\operatorname{card}(H)=\operatorname{card}(D \backslash C) \backslash H(=\operatorname{card}(D \backslash(C \cup H))=\operatorname{card}(D \backslash C)=\operatorname{card}(X)) .
$$

Let $K=\{F \subseteq D \backslash(H \cup C): \operatorname{card}(F \cup\{b\})=\operatorname{card}(X \backslash D)\}$, and consider the following claim:

Claim. For $F \in K$ we have $C \subseteq X \backslash(F \cup\{b\}) \in \mathbb{W}$. Suppose $F \in K$, so $F \subseteq D \backslash(H \cup C)$ so $H \subseteq X \backslash(F \cup\{b\}) \subseteq X$ thus $\operatorname{card}(X \backslash(F \cup\{b\}))=\operatorname{card}(X)=$ $\operatorname{card}(D)$ and $\operatorname{card}(X \backslash D)=\operatorname{card}(F \cup\{b\})=\operatorname{card}(X \backslash(X \backslash(F \cup\{b\})))$, therefore $X \backslash(F \cup\{b\}) \in \mathbb{W}$. Also $F \subseteq D \backslash(H \cup C)$ and $b \notin C$ show $C \subseteq X \backslash(F \cup\{b\})$.

Therefore

$$
\begin{aligned}
\eta: & K \rightarrow\{B \in \mathbb{W}: C \subseteq B\} \\
& F \mapsto X \backslash(F \cup\{b\})
\end{aligned}
$$

is well-defined and clearly one-to-one.

Thus $\operatorname{card}(K) \leq \operatorname{card}(\{B \in \mathbb{W}: C \subseteq B\}) \leq \operatorname{card}(\mathbb{W})$, however using $\operatorname{card}(D \backslash(H \cup$ $C))=\operatorname{card}(X \backslash\{b\})$ we have:

$$
\begin{aligned}
\operatorname{card}(\mathbb{W}) & \leq \operatorname{card}(\{E \subseteq X \backslash\{b\}: \operatorname{card}(E)=\operatorname{card}(X \backslash(D \cup\{b\}))\}) \\
& =\operatorname{card}(\{F \subseteq D \backslash(H \cup C): \operatorname{card}(F)=\operatorname{card}(X \backslash(D \cup\{b\}))\}) \\
& =\operatorname{card}(K)
\end{aligned}
$$


which leads to $\operatorname{card}(K)=\operatorname{card}(\{B \in \mathbb{W}: C \subseteq B\})=\operatorname{card}(\mathbb{W})$.

For $E \subseteq X$ with $E \approx C$ (so $b \notin E$ ), we have $D^{\prime}=(D \backslash C) \cup E \in \mathbb{W}$ and $\mathbb{W}=\left\{E \subseteq X \backslash\{b\}: \operatorname{card}(E)=\operatorname{card}\left(D^{\prime}\right) \wedge \operatorname{card}(X \backslash E)=\operatorname{card}\left(X \backslash D^{\prime}\right)\right\}$. Using a similar method described above, we have $\operatorname{card}(\{B \in \mathbb{W}: E \subseteq B\})=\operatorname{card}(\mathbb{W})$, thus $\mathbb{W}$ is a $C-(X, D, \operatorname{card}(\mathbb{W}))$ Top-design of type 1 .

$\left.a_{3}\right)$ In this case if $\mathbb{A}$ is a $C-(X, D, \lambda)$ Top-design of type 1 , then there exists $\bar{B} \in \mathbb{A}$ with $X \backslash\{b\} \subseteq B$ (since $C \approx X \backslash\{b\}$ ), which leads to $D=X \backslash\{b\}$, and $\mathbb{W}=\{X \backslash\{b\}\}$ is a $C-(X, D, 1)$ Top-design of type 1 .

b) Use the fact that if $b \notin D$, then for all $B \subseteq X$ with $D \approx B$ and $X \backslash D \approx X \backslash B$ we have $b \notin B$, and in particular $C \nsubseteq B$.

$\left.c_{1}\right)$ First suppose $\mathbb{A}$ is a $C-(X, D, \lambda)$ Top-design of type 1 and $D$ is finite, then for all subsets $H$ of $X$ with $\operatorname{card}(H)=\operatorname{card}(C)$, there exists $B \in \mathbb{A}$ with $H \subseteq B$, however we may assume $b \notin H$, using $b \in B$ we have $\operatorname{card}(H) \leq \operatorname{card}(B \backslash\{b\})=$ $\operatorname{card}(D)-1$. Hence $\operatorname{card}(C)+1 \leq \operatorname{card}(D)$. If $\operatorname{card}(C)+1=\operatorname{card}(D)$ then any subset of $X \backslash\{b\}$ with $\operatorname{card}(C)$ elements occurs in just one element of $\mathbb{A}$ and $\mathbb{A}=\{S \cup\{b\}: S \subseteq X \backslash\{b\} \wedge \operatorname{card}(S)=\operatorname{card}(C)\}$ now choose a subset $J$ of $X \backslash\{b\}$ with $\operatorname{card}(C)-1$ elements, then infinite elements of $\mathbb{A}$ contain $J \cup\{b\}(\approx C)$ which is in contradiction with $\lambda=1$, so $\operatorname{card}(C)+1<\operatorname{card}(D)$ and $\operatorname{card}(C)+2 \leq \operatorname{card}(D)$. In order to complete the proof, we have the following cases:

Case 1. $X$ is uncountable and $D$ is infinite. In this case choose infinite countable subset $I$ of $D \backslash\{b\}$. By the proof of $\left(\mathrm{a}_{2}\right)$ for

$$
\mathbb{W}_{-b}=\{E \subseteq X: E \approx D \backslash\{b\} \wedge X \backslash E \approx X \backslash(D \backslash\{b\})\}
$$

is a $I-\left(X, D \backslash\{b\}, \operatorname{card}\left(\mathbb{W}_{-b}\right)\right)$ Top-design of first type. We show $\mathbb{W}$ is a $C-$ $(X, D, \operatorname{card}(\mathbb{W}))$ Top-design of first type. Consider $H \subseteq X$ with $H \approx C$. There exists $J \subseteq X \backslash\{b\}$ with $H \backslash\{b\} \subseteq J$ and $J \approx I$ so

$$
\begin{aligned}
\operatorname{card}\left(\mathbb{W}_{-b}\right) & \geq \operatorname{card}\left(\left\{B \in \mathbb{W}_{-b}: H \backslash\{b\} \subseteq B\right\}\right) \\
& \geq \operatorname{card}\left(\left\{B \in \mathbb{W}_{-b}: J \subseteq B\right\}\right)=\operatorname{card}\left(\mathbb{W}_{-b}\right)
\end{aligned}
$$

therefore $\operatorname{card}\left(\left\{B \in \mathbb{W}_{-b}: H \backslash\{b\} \subseteq B\right\}\right)=\operatorname{card}\left(\mathbb{W}_{-b}\right)$. Considering bijection $\eta: \mathbb{W}_{-b} \rightarrow \mathbb{W}$, and $b \in \bigcap \mathbb{W}$ we have $\operatorname{card}(\{B \in \mathbb{W}: H \backslash\{b\} \subseteq B\})=\operatorname{card}(\{B \in$ $B \mapsto B \cup\{b\}$

$\left.\left.\mathbb{W}_{-b}: H \backslash\{b\} \subseteq B\right\}\right)=\operatorname{card}\left(\mathbb{W}_{-b}\right)=\operatorname{card}(\mathbb{W})$ which leads to $\operatorname{card}(\{B \in \mathbb{W}: H \subseteq$ $B\})=\operatorname{card}(\mathbb{W})$ and $\mathbb{W}$ is a $C-(X, D, \operatorname{card}(\mathbb{W}))$ Top-design of first type.

Case 2. $X, D$ and $X \backslash D$ are infinite countable. In this case we may suppose $X \backslash\{b\}=\left\{p_{n}: n \geq 1\right\}$ and $D=\left\{p_{2 n}: n \geq 1\right\} \cup\{b\}$ with distinct $p_{n}$ s. Let $\mathbb{A}=\left\{X \backslash\left\{p_{2 k+1}: k \geq s\right\}: s \geq 1\right\}$, then $\mathbb{A}$ is a $C-\left(X, D, \aleph_{0}\right)$ Top-design of type 1 , Case 3. $X$ and $D$ are infinite countable and $X \backslash D \neq \varnothing$ is finite. In this case $\mathbb{W}$ is infinite countable and a $C-\left(X, D, \aleph_{0}\right)$ Top-design of type 1 .

Case 4. $X=D$ is infinite countable. In this case $\mathbb{W}=\{X\}$ is a $C-(X, D, 1)$ Top-design of type 1 .

Case 5. $D$ is finite and $\operatorname{card}(C)+2 \leq \operatorname{card}(D)$. In this case card $(\mathbb{W})=\operatorname{card}(X)$ (since for infinite set $X$ we have $\operatorname{card}(X)=\operatorname{card}\left(\mathcal{P}_{\text {fin }}(X)\right.$ ), where $\mathcal{P}_{\text {fin }}(X)$ ) is the collection of all finite subsets of $X)$ and $\mathbb{W}$ is a $C-(X, D, \operatorname{card}(X))$ Top-design of type 1 .

$\underline{c_{2}}$ ) In this case by the proof of $\left(\mathrm{a}_{2}\right), \mathbb{W}$ is a $C \backslash\{b\}-(X, D \backslash\{b\}$, card(WW) Topdesign of type 1 , using $b \in \bigcap \mathbb{W}$, shows that $\mathbb{W}$ is a $C-(X, D$, card( $\mathbb{W}))$ Top-design of type 1 too.

$\left.\underline{c_{3}}\right)$ Use a similar method described in the proof of $\left(\mathrm{a}_{3}\right)$. 
Lemma 2.3. For nonempty subsets $C, D$ of $X, C$ can be embedded into $D$ if and only if

$$
\text { "C is finite or } b \notin C \backslash D \text { ", and "card }(C) \leq \operatorname{card}(D) \text { ". }
$$

Proof. Suppose $C$ can be embedded in $D$ and choose $E \subseteq D$ with $E \approx C$, so $\operatorname{card}(C)=\operatorname{card}(E) \leq \operatorname{card}(D)$. If $C$ is infinite and $b \in C$ then any subset of $X$ homeomorphic with $C$ contains $b$, thus $b \in E(\subseteq D)$ and $b \notin C \backslash D$.

Theorem 2.4. For nonempty subsets $C, D$ of $X$, there exist $\lambda$ and a $C-(X, D, \lambda)$, Top-design of type 2 if and only if $C$ can be embedded into $D$.

Proof. If we can not embed $C$ into $D$ it's evident that there is not any $C-(X, D, \lambda)$, Top-design of type 2 .

Conversely suppose $C$ can be embedded in $D$, so by Lemma 2.3 card $(C) \leq \operatorname{card}(D)$ and "C is finite or $b \notin C \backslash D$ ". Let $\mathbb{L}=\{E \subseteq X: E \approx D\}$. We have the following cases:

- $\operatorname{card}(C) \leq \operatorname{card}(D)$ and $C$ is finite. In this case $\mathbb{L}$ is a $C-(X, D, \lambda)$ Top-design of type 2 with:

$$
\lambda=\left\{\begin{array}{lc}
1 & \operatorname{card}(C)=\operatorname{card}(D), \\
\operatorname{card}(\{E \subseteq X: \operatorname{card}(E)=\operatorname{card}(D)\}) & \text { otherwise }
\end{array}\right.
$$

For this aim use the fact that $\eta:\{E \subseteq X \backslash C: \operatorname{card}(E)=\operatorname{card}(D)\} \rightarrow\{E \subseteq X$ : $\operatorname{card}(E)=\operatorname{card}(D)\}$ with $\eta(E)=E \cup C$ is bijective.

- $\operatorname{card}(C)=\min (\operatorname{card}(C), \operatorname{card}(D))<\operatorname{card}(X)$ and $b \notin C \backslash D$. In this case by Theorem 2.2 there exists $\lambda$ and $C-(X, D, \lambda)$, Top-design of type 1 , so it is a $C-(X, D, \lambda)$, Top-design of type 2 too.

- $\operatorname{card}(C)=\min (\operatorname{card}(C), \operatorname{card}(D))=\operatorname{card}(X)$ and $b \notin C \backslash D$. In this case $\mathbb{A}=$ $\{(X \backslash\{b\}) \cup(D \cap\{b\})\}$ is a $C-(X, D, 1)$ Top-design of type 2 .

Theorem 2.5. Regarding 3rd type of Top-designs for nonempty subsets $C, D$ of $X$, there exist $\lambda$ and a $C-(X, D, \lambda)$, Top-design of type 3 if and only if $b \notin C \backslash D$, $\operatorname{card}(C \backslash\{b\}) \leq \operatorname{card}(D \backslash\{b\})$ and $\operatorname{card}(X \backslash(D \cup\{b\})) \leq \operatorname{card}(X \backslash(C \cup\{b\}))$.

Proof. Let $\mathbb{W}=\{E \subseteq X: E \approx D \wedge X \backslash E \approx X \backslash D\}$. If $\mathbb{A}$ is a $C-(X, D, \lambda)$, Top-design of type 3 , then $\mathbb{A} \subseteq \mathbb{W}$ and we have the following cases:

Case 1. $b \in C \backslash D$. In this case for all $E \in \mathbb{A}(\subseteq \mathbb{W})$, we have $b \notin E$ and $C \nsubseteq E$ thus $\mathbb{A}$ is not a $C-(X, D, \lambda)$, Top-design of type 3 .

Case 2. $\operatorname{card}(C \backslash\{b\})>\operatorname{card}(D \backslash\{b\})$, and " $b \in C \cap D$ or $b \notin C \cup D$ ". In this case we have $\operatorname{card}(C)>\operatorname{card}(D)$ so we can not embed $C$ into $D$ and it's evident that there is not any $C-(X, D, \lambda)$, Top-design of type 3 .

Case 3. $\operatorname{card}(C \backslash\{b\})>\operatorname{card}(D \backslash\{b\}), b \in D \backslash C$. In this case for all $B \in \mathbb{A}, b \in B$ and $\operatorname{card}(C)=\operatorname{card}(C \backslash\{b\})>\operatorname{card}(D \backslash\{b\})=\operatorname{card}(B \backslash\{b\})$ so $C \nsubseteq B \backslash\{b\}$ and $C \not \subset B$ so $\mathbb{A}$ is not a $C-(X, D, \lambda)$, Top-design of type 3 .

Case 4. $\operatorname{card}(X \backslash(D \cup\{b\}))>\operatorname{card}(X \backslash(C \cup\{b\}))$. In this case for all $E \in \mathbb{W}$ we have $\operatorname{card}(X \backslash(E \cup\{b\}))>\operatorname{card}(X \backslash(C \cup\{b\}))$, thus $X \backslash(E \cup\{b\}) \nsubseteq X \backslash(C \cup\{b\})$ and $C \nsubseteq E$, so there is not any $C-(X, D, \lambda)$, Top-design of type 3 .

Considering the above cases $b \notin C \backslash D, \operatorname{card}(C \backslash\{b\}) \leq \operatorname{card}(D \backslash\{b\})$ and $\operatorname{card}(X \backslash$ $(D \cup\{b\})) \leq \operatorname{card}(X \backslash(C \cup\{b\}))$.

Conversely, suppose $b \notin C \backslash D, \operatorname{card}(C \backslash\{b\}) \leq \operatorname{card}(D \backslash\{b\})$ and $\operatorname{card}(X \backslash(D \cup$ $\{b\})) \leq \operatorname{card}(X \backslash(C \cup\{b\}))$, then $\mathbb{W}$ is a $C-(X, D, \lambda)$, Top-design of type 3 for 
$\lambda=\operatorname{card}(\{E \in \mathbb{W}: C \subseteq E\})$ (note that for $F \subseteq X$ with $F \approx C$ and $X \backslash F \approx X \backslash C$, the map $\{E \in \mathbb{W}: F \subseteq E\} \rightarrow\{E \in \mathbb{W}: C \subseteq E\}$ is bijective).

$$
E \mapsto(E \backslash F) \cup C
$$

Theorem 2.6. For nonempty subsets $C, D$ of $X$, there exist $\lambda$ and a $C-(X, D, \lambda)$, Top-design of type 4 if and only if $C$ can be embedded into $D$.

Proof. If $C$ can be embedded into $D$, then there exist $\lambda>0$ and a $C-(X, D, \lambda)$ Top-design of type 2 like $\mathbb{A}$ by Theorem2.4, so $\mathbb{A}$ is a $C-(X, D, \lambda)$ Top-design of type 4 too.

Conversely, it's evident that if $\mathbb{A}$ is a Top-design of type $i$ (for $i=1,2,3,4$ ), then there exists $E \in \mathbb{A}$ with $C \subseteq E$, using $E \approx D$ leads us to the fact that $C$ can be embedded into $D$.

Theorem 2.7. For nonempty subsets $C, D$ of $X$ the following statements are equivalent:

- there is not any $C-(X, D, \lambda)$, Top-design of type 2 ,

- there is not any $C-(X, D, \lambda)$, Top-design of type 4 ,

- "C is infinite and $b \in C \backslash D$ ", or "card $(C)>\operatorname{card}(D)$ ",

- $C$ can not be embedded into $D$.

Proof. Theorems 2.4, 2.6 and Lemma 2.3.

\section{ACKNOWLEDGEMENT}

The authors are grateful to the research division of the University of Tehran for the grant which supported this research.

\section{REFERENCES}

[1] I. Anderson, Combinatorial designs: construction methods, Ellis Horwood Ltd., 1990.

[2] F. Ayatollah Zadeh Shirazi and M. Bagherian, Generalized-designs, Missouri Journal of Mathematical Sciences, 23, no. 1 (2011), 27-47.

[3] P. J. Cameron, and B. S. Webb, What is an infinite design?, Journal of Combinatorial Designs, 10:2 (2002), 79-91.

[4] L. A. Steen and J. A. Seebach Jr., Counterexamples in topology, New York-Montreal, Holt, Rinehart and Winston Inc., 1970.

Mehrnaz Pourattar, Islamic Azad University, Science and Research Branch, Tehran, Iran (e-mail: mpourattar@yahoo.com)

Fatemah Ayatollah Zadeh Shirazi, Faculty of Mathematics, Statistics and Computer Science, College of Science, University of Tehran, Enghelab Ave., Tehran, Iran (e-mail: fatemah@khayam.ut.ac.ir) 